\title{
Elements of string cosmology
}

\section{Citation}

Tseytlin, A.A., and C. Vafa. 1992. "Elements of String Cosmology." Nuclear Physics B 372 (1-2): 443-66. https://doi.org/10.1016/0550-3213(92)90327-8.

\section{Permanent link}

http://nrs.harvard.edu/urn-3:HUL.InstRepos:41385052

\section{Terms of Use}

This article was downloaded from Harvard University's DASH repository, and is made available under the terms and conditions applicable to Other Posted Material, as set forth at http:// nrs.harvard.edu/urn-3:HUL.InstRepos:dash.current.terms-of-use\#LAA

\section{Share Your Story}

The Harvard community has made this article openly available.

Please share how this access benefits you. Submit a story.

Accessibility 
HUTP-91/A049

JHU-TIPAC-910028

\title{
Elements of String Cosmology
}

\author{
A. A. Tseytlin ${ }^{\dagger}$ \\ Department of Physics and Astronomy \\ The Johns Hopkins University \\ Baltimore, Maryland 21218, USA \\ C. Vafa \\ Lyman Laboratory of Physics \\ Harvard University \\ Cambrdige, MA 02138, USA
}

\begin{abstract}
Aspects of string cosmology for critical and non-critical strings are discussed emphasizing the necessity to account for the dilaton dynamics for a proper incorporation of "large - small" duality. This drastically modifies the intuition one has with Einstein's gravity. For example winding modes, even though contribute to energy density, oppose expansion and if not annihilated will stop the expansion. Moreover we find that the radiation dominated era of the standard cosmology emerges quite naturally in string cosmology. Our analysis of non-critical string cosmology provides a reinterpretation of the (universal cover of the) recently studied two dimensional black hole solution as a conformal realization of cosmological solutions found previously by Mueller.
\end{abstract}

$9 / 91$

$\dagger$ On leave of absence from the Department of Theoretical Physics, P. N. Lebedev Physics Institute, Moscow 117924, USSR. Address from October 1, 1991 : DAMTP, University of Cambridge, Cambridge CB3 9EW and Trinity College, Cambridge, CB2 1TQ, Cambridge, United Kingdom. 


\section{Introduction}

Despite tremendous progress in our understanding of fundamental strings in the past decade, we are still very far from a single quantitative prediction to be observed in experiments! The main reason for this unsatisfactory state of affairs is that the natural scale in which the stringy effects become important (Planck scale) is much smaller than the scale we can probe in high energy scattering experiments. However, it has been known for a while that cosmology provides a window to test fundamental physics, since the scales of particle physics theories become relevant as the universe evolves in its early history. It is our belief that the most likely area for a confrontation between string theory and experiments is through extracting cosmological consequences of string theory which may be measurable today. Many of the riddles of astrophysics, for example 'dark matter' and large scale structure of the universe may turn out to be related to fundamental strings. It is with such a program in mind that we study string cosmology in this paper.

To see the effects of strings we have to trace back the universe to its earliest times. Here already string theory provides us with a surprise: strings in very small spaces behave the same way as strings in very large spaces $[1,2]$. This is true at least in the simplest known cases (toroidal backgrounds) and is suspected to be a general principle of string theory, because strings should be 'blind' to scales smaller than their own natural scale. Thus the universe does not start at a zero size, as that would be equivalent to infinitely large universe, rather we should start with a universe of the size of the string scale, i.e., the Planck length. This $a \rightarrow 1 / a$ duality (in Planck units) already hints of a major modification to Einstein equations at early times, as ordinary theory of general relativity is not invariant under such a transformation. It has been suggested that, roughly speaking, string theory necessitates the existence of two kinds of matter fields, "dual" to each other. For large radii, one type becomes massless and dominates, while for small radii the other type becomes massless and dominates. This suggests an effective "doubling" of the number of space dimensions (see e.g. [2,3]). Needless to say to have an exact treatment of dynamics in 
such a situation is beyond our scope at the present time. This was one of the main reasons why the cosmological ideas in [2] were rather difficult to test, as the dynamical equations governing string cosmology were not properly understood.

It turns out that it is possible to introduce a consistent dynamics which describes slowly evolving states of strings in a duality invariant manner. This involves, in particular, taking into account the dilaton field which transforms under the duality [4-7].

It is a fundamental consequence of string theory that the gravitational interaction is effectively described not by the metric alone but by the coupled system of the metric and the dilaton. Dilaton of course should get a mass to avoid a scalar component of gravitational interaction of massive bodies but this probably happens at a later stage of evolution of the Universe. That implies that one cannot not ignore the dilaton dynamics at early "stringy" phases of cosmological evolution. As we shall see, the assumption that dilaton is constant is in general inconsistent with the cosmological equations describing evolution of matter with the "stringy" equation of state1.

The main body of this paper is devoted to the discussion of the duality invariant cosmological equations describing the evolution of the "scales" of the metric and the dilaton. We shall study their solutions emphasizing departures from Einstein's gravity theory. For example, we will find that a constant energy density not only does not lead to inflation, but it rather slows and eventually stops expansion2. Similarly, we will discover that if

1 Dilaton dynamics was ignored in most previous discussions of string cosmology at finite temperature (see e.g. [8] and refs. there). Dilaton was not set equal to constant in ref.[9] where, however, the space was not taken to be compact and hence many of the stringy effects, and in particular duality invariance was not discussed.

2 We shall describe the expansion/contraction in terms of the original unrescaled ( $\sigma$-model" ) metric. Though the effective gravitational constant is then time dependent, this is the metric string directly interacts with and hence the one measured by stringy "rods". Similar point of view was expressed in [10]. The use of this metric is appropriate at early phase of cosmological evolution when dilaton is still massless (for a discussion of a possible inflation in string cosmology described in terms of the rescaled metric after the dilaton already got mass see [11]). Let us note also that the use of the "unrescaled" metric is particularly natural in the duality symmetric case since it has a simple transformation law under the duality. 
the winding modes (by "winding modes" we mean a more general notion of stringy states whose masses grow with a growing scale of the universe) are not annihilated (which may happen if they are not in thermal equilibrium) they halt the expansion. Using our equations we can study some simple aspects of the cosmological scenario proposed in [2] in which the three dimensionality of extended space was suggested to be a consequence of string cosmology.

The equations we consider apply to both critical and non-critical strings and we study them both in the presence and in the absence of matter. The simplest solution corresponding to non-critical strings was first proposed by Myers [12] as a way to change the dimension of target space in string theory and was then used for construction of cosmological solutions in [13]. These solutions were further extended by Mueller [14] who found non-trivial cosmological solutions for which the radii of the toroidal universe change with time. We will show that specializing his solution to $d=2$ one recovers (a region of) the two dimensional black-hole solution, which has been found to be related (in the leading order approximation) to a conformal theory $[15]^{3}$. We find that the more natural setup to discuss $\mathrm{d}=2$ cosmology is to go to an infinite fold cover of $S L(2, R) / U(1)$. This will avoid getting timelike loops and give us infinitely many universes parametrized by an integer $n$, such that the future of particles in the universe $n$ could be in any universe $m \geq n$. The exact metric suggested for the $\mathrm{d}=2$ Euclidean black hole [17] which leads to a puzzle within a black-hole interpretation seems perfectly consistent once we use such a cosmological interpretation (corresponding to the universal cover of $S L(2, R) / U(1)$ ).

The organization of this paper is as follows. In section 2 we derive the basic equations of string cosmology in the adiabatic approximation and discuss their duality invariance property. In section 3 we specialize to critical strings and consider some basic elements of string cosmology by studying the solutions of these equations. We find a simple intuitive

3 As was already pointed out in [5], the euclidean continuation of the $d=2$ Myers solution coincides with the euclidean solution independently discovered in [16]. 
way to think about the solutions based on the analogy with a mechanical model of a particle rolling on a potential under the influence of a damping force. In section 4 we concentrate on some aspects of non-critical string cosmology and its relation to the $\mathrm{d}=2$ black hole. We also briefly discuss how string one loop effects might modify the non-critical cosmological solutions. In section 5 we present our conclusions. In appendix A some aspects of the solutions to our cosmological equations are worked out.

\section{General Structure of Cosmological Equations}

Let us consider strings propagating in a large universe of spatial dimension $N$. If $N$ is not the critical spatial dimension of strings (i.e., if $N<25(9)$ for (super)strings), we could have one of two possibilities: either we have some $N_{c}$ of compact internal dimensions which make up the rest of the spatial dimensions which we take to be static and to correspond to conformal theories on the worldsheet, or we are dealing with a "non-critical" string. In other words, if we set

$$
c=\frac{2}{3}\left(d_{c}-N-N_{c}\right)
$$

where $d_{c}$ is the critical spatial dimension, in the first case $c=0$, and in the second case $c \neq 0$. For simplicity we take the $N$-dimensional space to be a periodic box (torus) of lengths $a_{i}=\exp \lambda_{i}$ ( $i$ runs from 1 to $N$ ). However, many of the ideas developed in this paper can easily be extended to more general cases.

It is a well known principle in string theory that as long as we concentrate on slowly varying fields, we can hope to describe the global aspects of the dynamics of the theory reasonably well by concentrating on massless modes, and keeping only the leading derivative terms ('adiabatic' approximation). The types of massless modes depend on a particular choice of vacuum backgrounds strings are propagating in, and on whether we are considering bosonic strings, superstrings, or heterotic strings. But they always include a gravitational field and a dilaton (for simplicity we shall ignore the antisymmetric tensor 
field). If the radii are large compared to the string scale the leading order terms in the low energy expansion of the tree level gravitational-dilaton effective action of the closed string theory are [18] (we shall absorb the gravitational coupling constant into $\phi$ and set $\alpha^{\prime}=1$ )

$$
S_{0}=-\int d^{N+1} x \sqrt{-G} \mathrm{e}^{-2 \phi}\left[c+R+4(D \phi)^{2}\right]
$$

Note that $\mathrm{e}^{\phi}$ plays the role of coupling 'constant' in string theory. The first question we would like to address is whether we should trust this action at all for Planckian size spatial dimensions, i.e., when $\lambda_{i} \sim 0$. The answer is no, if we wish to take all the modes in the above action seriously. The above action manifestly breaks the duality symmetry of string theory. The reason is that when we approach Planckian size universes the momentum modes (which correspond to inhomogeneity in the metric and dilaton fields) become massive, and the winding modes which wrap around the box and were ignored previously will have to be taken into account, as they have mass comparable to that of the momentum modes. This 'doubling of the space' seems very difficult to deal with, and on top of that we have to remember that the oscillator (higher level) modes are also going to become important since they will be of the same energy as the momentum modes.

However, the following observation allows us to still take part of the above action seriously. The point is that the space independent parts of the above fields have zero energy independent of the size of the universe, and thus we can naturally concentrate on them, setting the other Planckian modes to zero. Note in particular that these spatially independent modes are common to both the momentum and winding excitations, so truncating to these modes alone will be consistent with duality. Therefore we should expect that even though the above action naively seems to pick momentum modes over winding modes, i.e., the space over the dual space, concentrating on spatially constant fields restores the duality symmetry. Thus we should trust the above action as far as the overall scales of

\footnotetext{
4 The volume of the internal space, if there is any, can be gotten rid of by a shift in $\phi$.
} 
the universe are concerned and not so much as a description of inhomogeneities of the universe. And in addition we should assume that the fields are varying slowly with time.

Let us see how the duality invariance $a_{i} \rightarrow 1 / a_{i}$ is restored in the above action once we concentrate on zero modes. Let us therefore consider the metric and the dilaton field of the form

$$
\begin{gathered}
d s^{2}=-d t^{2}+\sum_{i=1}^{N} a_{i}^{2}(t) d x_{i}^{2} \\
a_{i}=\mathrm{e}^{\lambda_{i}(t)}, \quad \phi=\phi(t)
\end{gathered}
$$

It is useful to introduce the "shifted" (and rescaled by 2) dilaton field $\varphi$ which absorbs the space volume factor

$$
\varphi \equiv 2 \phi-\sum_{i=1}^{N} \lambda_{i}, \quad \sqrt{-G} \mathrm{e}^{-2 \phi}=\mathrm{e}^{-\varphi} .
$$

Then the action becomes

$$
S_{0}=-\int d t \mathrm{e}^{-\varphi} \sqrt{-G_{00}}\left[c-G^{00} \sum_{i=1}^{N} \dot{\lambda}_{i}^{2}+G^{00} \dot{\varphi}^{2}\right]
$$

We have kept $G_{00}$ as we need to vary the action with respect to it in order to get the full set of equations of motion ( in the equations of motion $G_{00}$ is set to -1 ). Dot denotes the time derivative. The above action is manifestly invariant under the duality transformation $[4-6]$

$$
\lambda_{i} \rightarrow-\lambda_{i} \quad, \quad \phi \rightarrow \phi-\lambda_{i} \quad, \quad \varphi \rightarrow \varphi
$$

(here $i$ is any number among $1, . ., N$; more general duality transformations are obtained by combining these basic ones).

The above action is written in the absence of stringy "matter". To discuss cosmology it is important to incorporate matter into the system. Assuming that the effective string coupling is small let us take the matter to be a gas of (free) string modes in a thermal equilibrium at the temperature $\beta^{-1}$. Then matter contribution to the action is represented by

$$
S_{m}=\int d t \sqrt{-G_{00}} F\left(\lambda_{i}, \beta \sqrt{-G_{00}}\right)
$$


Here $F$ is the (one loop) free energy and can be represented in terms of the one loop string partition function in a torus of radii $\mathrm{e}^{\lambda_{i}}$ and periodic Euclidean time of perimeter $\beta \sqrt{-G_{00}}$ , $Z=-\beta F$. The adiabaticity assumption implies that we can replace constant radii and $\beta$ by functions of time. Taking the full action

$$
S=S_{0}+S_{m}=-\int d t \sqrt{-G_{00}}\left[\mathrm{e}^{-\varphi}\left(c-G^{00} \sum_{i=1}^{N} \dot{\lambda}_{i}^{2}+G^{00} \dot{\varphi}^{2}\right)-F\left(\lambda_{i}, \beta \sqrt{-G_{00}}\right)\right]
$$

and varying it with respect to $\lambda_{i}, \phi$ and $G_{00}$ and we find the following equations 5

$$
\begin{gathered}
c-\sum_{i=1}^{N} \dot{\lambda}_{i}^{2}+\dot{\varphi}^{2}=\mathrm{e}^{\varphi} E, \\
\ddot{\lambda}_{i}-\dot{\varphi} \dot{\lambda}_{i}=\frac{1}{2} \mathrm{e}^{\varphi} P_{i}, \\
\ddot{\varphi}-\sum_{i=1}^{N} \dot{\lambda}_{i}^{2}=\frac{1}{2} \mathrm{e}^{\varphi} E,
\end{gathered}
$$

where

$$
\begin{gathered}
E=-2 \frac{\delta S_{m}}{\delta G_{00}}=F+\beta \frac{\partial F}{\partial \beta}, \\
P_{i}=-\frac{\delta S_{m}}{\delta \lambda_{i}}=-\frac{\partial F}{\partial \lambda_{i}} .
\end{gathered}
$$

$E$ is the total energy of the matter and $P_{i}$ is the pressure in the i-th direction times the volume. These equations imply a modified conservation law for the energy (following from the invariance under reparametrizations of time )

$$
\dot{E}+\sum_{i=1}^{N} \dot{\lambda}_{i} P_{i}=0 .
$$

Since $F=F(\lambda(t), \beta(t))$ this is equivalent to the conservation of the entropy $\mathcal{S}=\beta^{2} \partial F / \partial \beta$, i.e. our matter is indeed evolving adiabatically. This means that for a given radius determined by $\lambda_{i}$ the temperature $\beta^{-1}$ adjusts itself so that $\mathcal{S}$ remains constant. Solving the

5 Similar equations were discussed in [9] and also in [6] where the matter was represented by the energy-momentum tensor of a macroscopic string. Even though we derived the above equations using the canonical ensemble, they are valid even when the canonical ensemble description breaks down (as is the case near Hagedorn temperature) and we have to use the more fundamental microcanonical ensemble. 
adiabaticity condition we can express $\beta$ in terms of $\lambda_{i}$ and hence represent $E$ as a function of $\lambda_{i}$ alone,

$$
E(\lambda)=E(\lambda, \beta(\lambda))
$$

In what follows we shall consider only this new function $E(\lambda)$. Since entropy is not changing we find

$$
P_{i}=-\frac{\partial E(\lambda)}{\partial \lambda_{i}}
$$

Note that the time derivative of (2.3) vanishes once we use (2.4) and (2.5), and so the non-trivial content of equation (2.3) is that it fixes the constant of integration in terms of $c$. The above equations are duality invariant since $F$ is invariant under $\lambda \rightarrow-\lambda$ for a fixed temperature $\left(E(\lambda)\right.$ and $\beta(\lambda)$ are also invariant and $P_{i}$ changes sign under the duality).

Rewritten in terms of the original dilaton field $\phi$ equations $(2.3)-(2.5)$ take the form

$$
\begin{gathered}
c-\sum_{i=1}^{N} \dot{\lambda}_{i}^{2}+\left(2 \dot{\phi}-\sum_{i=1}^{N} \dot{\lambda}_{i}\right)^{2}=\mathrm{e}^{2 \phi} \rho \\
\ddot{\lambda}_{j}-\left(2 \dot{\phi}-\sum_{i=1}^{N} \dot{\lambda}_{i}\right) \dot{\lambda}_{j}=\frac{1}{2} \mathrm{e}^{2 \phi} p_{j} \\
2 \ddot{\phi}-\sum_{i=1}^{N} \ddot{\lambda}_{i}-\sum_{i=1}^{N} \dot{\lambda}_{i}^{2}=\frac{1}{2} \mathrm{e}^{2 \phi} \rho
\end{gathered}
$$

where $\rho=E / V, p_{i}=P_{i} / V$ and $V=\exp \sum \lambda_{i}$ is the space volume. To compare these equations with standard cosmological equations in which the dilaton is ignored consider setting $\phi$ (which is related to the coupling constant) to be constant. Then the equation (2.7) takes the well known form (with flat space),

$$
\left(\frac{\dot{a}}{a}\right)^{2}=\dot{\lambda}^{2}=\frac{-c}{N^{2}-N}+G \rho
$$

for some constant $G$, where $-c /\left(N^{2}-N\right)$ plays the role of the cosmological constant 6 . However, it is easy to see that if $c=0$ a solution with $\phi=$ const is consistent with all the

6 It is amusing to note that in this setup the problem of the vanishing cosmological constant seems to be related to the question of being on or off-criticality in string theory. This connection (which was already mentioned in the past) seems worth further study. 
three equations only if

$$
\sum_{i=1}^{N} p_{i}=\rho
$$

i.e., for a matter with the vanishing energy momentum trace, as is effectively the case for a gas of massless particles in a thermodynamical equilibrium. This "radiation -type" condition is not satisfied in a high temperature phase of string thermodynamics and is the reason for stringy departure from Einstein's gravity.

Let us discuss some general aspects of equations (2.3), (2.4) and (2.5). It is important to notice that the equations for $\lambda_{i}(2.4)$ are the same as that of a point particle in the presence of a time dependent potential $\frac{1}{2} \mathrm{e}^{\varphi} E(\lambda)$ and damped or boosted (depending on whether $\dot{\varphi}$ is negative or positive) by a dilaton "friction" force. Note that within this mechanical interpretation the "integration constant" $c$ in (2.3) plays the role of a fixed energy of the system.

Also, the equation for $\varphi$ can be summarized by subtracting equation (2.5) from (2.3) and defining $y=\mathrm{e}^{-\varphi}$, which leads to

$$
\ddot{y}+c y=\frac{1}{2} E
$$

This equation for $y$ can be interpreted as that for the endpoint of a spring, with spring constant $c$, and with the external force $\frac{1}{2} E(\lambda)$. These interpretations allow us to develop an intuitive picture for the nature of the solutions to our equations. As it turns out, the behavior of solutions will be rather different depending on whether or not we are describing critical strings, i.e., whether or not $c=0$.

\section{Critical String Cosmology}

This case corresponds to setting $c=0$. The important qualitative simplification that happens in this case is that since $E$ is positive (assuming no Casimir - like negative contribution to energy) from equation (2.3) we conclude that $\dot{\varphi}$ can never become zero. In 
other words, $\dot{\varphi}$ never changes sign, and thus it provides a damping or a boosting effect in equation (2.4) for all time. We will consider the damping case $\dot{\varphi}<0$; the boosting case can be obtained by time reversal. A strong reason to consider $\dot{\varphi}<0$ is that otherwise the boosting effect of dilaton on $\lambda$ will invalidate the adiabatic approximation used in the derivation of the above equations. Also, growing $\varphi$ together with expanding $\lambda$ implies the growth of the effective coupling $\exp \phi$ in contradiction with a weak coupling assumption.

From equation (2.5) it follows that $\ddot{\varphi}>0$ and thus $\dot{\varphi}$ is growing in time. Since $\dot{\varphi}$ is negative and it will never cross zero we conclude that (as long as $E \neq 0$ ) $\dot{\varphi}$ continues growing towards 0 and approaches 0 as $t \rightarrow \infty$.

For simplicity let us consider the isotropic case, i.e. assume that all $\lambda_{i}$ are equal to each other and denoted by $\lambda$ so that $a=\exp \lambda$ is the cosmological scale. The equations we get can be written as

$$
\begin{gathered}
c-N \dot{\lambda}^{2}+\dot{\varphi}^{2}=\mathrm{e}^{\varphi} E, \\
\ddot{\lambda}-\dot{\varphi} \dot{\lambda}=\frac{1}{2} \mathrm{e}^{\varphi} P, \\
\ddot{\varphi}-N \dot{\lambda}^{2}=\frac{1}{2} \mathrm{e}^{\varphi} E,
\end{gathered}
$$

where $P=-N^{-1} \partial E / \partial \lambda$ and for critical strings we simply set $c=0$ in (3.1).

In order to solve this system of equations we will need to specify $E(\lambda)$ as well as provide the initial conditions for $\lambda, \varphi$ and $\dot{\varphi} . E(\lambda)$ encodes string thermodynamics by specifying how the total energy in the box has to change as a function of $\lambda$ in order to keep the entropy constant. Motivated by string cosmology, the function $E(\lambda)$ was introduced and studied using string thermodynamics in [2] ( some properties of $E(\lambda)$ were further clarified in [19], see also [20] ). Its basic structure is indicated in fig. 1.

This function is duality symmetric under $\lambda \rightarrow-\lambda$. Its structure is well understood near $\lambda \sim 0$ and for very large $\lambda$. In the first region near $\lambda \sim 0$, which we call the Hagedorn region, the temperature of the strings is very close to the Hagedorn temperature and the energy is almost independent of $\lambda$. It is given by $E=T_{H} \mathcal{S}$ where $T_{H}$ is the 
Hagedorn temperature (fixed for a given string theory) and $\mathcal{S}$ is the total entropy. For large enough $\lambda$ temperature drops significantly compared to the Hagedorn temperature, and the massive modes of string go out of equilibrium so that we are left with the massless modes. In this 'radiation dominated' region we thus have the usual relation of how $E$ depends on the radius of the universe for a gas of massless particles. In fact, the temperature drops as $1 / a=\exp (-\lambda)$ and $E \propto T^{N+1} a^{N} \propto a^{-1}$. In other words $E=C \mathrm{e}^{-\lambda}$ where $C$ is determined by $\mathcal{S}$ and the number of massless modes. The behaviour of $E$ in the intermediate region between the Hagedorn region and the radiation dominated region is only partially understood but it has been shown that $E$ is monotonically decreasing (in the Hagedorn regime) with increasing $|\lambda|[19]$, and it is natural to believe that this is true for all $\lambda$. So if we start with the initial conditions that $\dot{\varphi}<0$ the decreasing of the potential $E$ with increasing $|\lambda|$ will force $\lambda$ to slide down the potential towards increasing values of $|\lambda|$, until we emerge at the radiation dominated era. The universe will continue its expansion, and in fact it will approach the radiation dominated era of the standard cosmology. In other words, independently of the details of $E$, or the initial conditions, one finds that $2 \phi=\varphi+N \lambda$, which determines the string coupling constant, rapidly approaches a constant and the radius ultimately grows as $a \sim A t^{\frac{2}{N+1}}$ for large $t$, as is the case for standard radiation dominated cosmology. This is discussed in appendix A.

What determines $N$, the number of extended spatial dimensions? One idea to explain why $N \leq 3$ was suggested in [2]. It was based on the possibility that if the space expands in more than three dimensions, the assumption of maintaining thermal equilibrium may not be a good one, as for example the winding modes of strings will have a hard time finding and annihilating one another. Thus they would fall out of equilibrium and will be around. It was suggested there that if winding modes are around this may stop expansion. The universe would not expand until it learns the lesson that it is only possible to expand in three or smaller number of dimensions.

Let us note that Einstein's theory of gravity leaves us uncomfortable with the above suggestion that winding modes may prevent expansion. Recall that cosmological expansion 
rate for a flat universe with vanishing cosmological constant satisfies $\dot{\lambda}^{2}=G \rho$ in FRW cosmologies. In particular any form of matter, since it contributes to $\rho$, helps accelerate expansion. This in particular implies that the winding modes accelerate expansion in standard gravity theory, rather than prevent expansion. On the other hand the idea in [2] was based on duality which states that what usual matter does for the size of the universe (i.e. accelerates expansion) the dual matter (the winding states) should do for the dual size of the universe (i.e., accelerate contraction). This was a puzzle which was not fully resolved in [2]. We will now see that our equations resolve this paradox by showing that they drastically differ from Einstein's equations in such cases and indeed are consistent with the idea that winding modes slow down and ultimately stop the expansion.

If the winding modes are around the energy $E$ is going to grow with $\lambda$, as larger $\lambda$ mean bigger boxes and thus more stretched winding strings with higher energies. The mass of the winding states will increase with $\lambda$ as $\exp \lambda$. $E$ growing with $\lambda$ means that $\ddot{\lambda}<0$ as $\lambda$ will have to climb the potential $E$. So this slows the expansion and in fact ultimately stops it as it is shown in appendix $A$. More generally, it is shown there that if we take for large $\lambda$

$$
E \propto \exp (\alpha \lambda)
$$

then for $\alpha$ positive the universe reaches a maximum size and then contracts back. Constant matter energy density corresponds to $\alpha=N$ and it prevents expansion even more strongly! So we conclude that dilaton suppresses the inflationary mechanism based on constant energy densityl. If $\alpha$ is negative the universe expands forever (as is the case for the radiation dominated era where $\alpha=-1$ ).

The duality symmetry of $E$ implies that while the winding modes (providing the growth of $E$ at large $\lambda$ ) oppose expansion, the momentum modes (providing the growth of

7 As was already noted in the Introduction, this does not rule out the possibility of inflation at a later stage of evolution when dilaton gets a mass and it is appropriate to use the redefined metric. 
$E$ at large negative $\lambda$ ) oppose contraction. As a result, in the absence of equilibrium the universe sees the effective $E$ represented as a dashed curve in fig. 1 and the radius of the Universe will be oscillating between maximal and minimal values.

So the basic picture one is led to is a universe which oscillates in many directions for a while around the Planck scale (maybe within a few orders of magnitude), until by coincidence it starts expanding in smaller number of directions (the most likely one being the largest possible dimension consistent with maintaining equilibrium, namely 3 ). Then it expands forever and we find ourselves in the radiation dominated era of the standard cosmology. This may also explain the large entropy problem. The entropy may be large because during this "trial and error" period of the early universe, as the strings were out of equilibrium we would generate ( if this process takes long ) a lot of entropy. In other words, the large entropy problem may be related to having an 'old' universe.

An important issue to resolve in string cosmology is to explain the absence of massless dilatons at the present time. One should expect that somehow a potential is generated for the dilaton and it will have to sit at its minimum picking up a mass. The mechanism of how this precisely should happen in string theory is not known yet and is the biggest gap in connecting string cosmology to observable cosmology. This is presumably related to the fundamental question of how supersymmetry breaking takes place in string theory while maintaining vanishing cosmological constant.

\section{Non-critical String Cosmology}

The case of non-critical string cosmology is an analog of non-vanishing cosmological constant case of cosmology in standard theory of gravity. We shall consider the case of $c>0$. Cosmological solutions for the toroidal space and non-zero $c$ in the absence of matter ( $E=0$ ) has been previously studied in [14]. One obtains from (2.11)( setting $E=0)$

$$
y=\mathrm{e}^{-\varphi}=A \sin 2 b t, \quad b^{2}=c / 4 .
$$


From (2.4) ( with $P_{i}=0$ ) one learns that

$$
\dot{\lambda}_{i}=k_{i} \mathrm{e}^{\varphi} \quad, \quad k_{i}=\text { const },
$$

which can be easily integrated to yield

$$
\lambda_{i}=\lambda_{i 0}+q_{i} \ln \tan b t,
$$

subject to the condition resulting from equation (2.3)

$$
\sum_{i=1}^{N} q_{i}^{2}=1
$$

These solutions can also be extended to $c \leq 0$ [14]. Note that for $c>0$ these solutions are singular, i.e., the fields blow up at finite time. In fact, as it is easy to see from (2.11), the oscillatory nature of solution forces $y$, which should be positive, to cross zero in finite time ( so that $\varphi$ blows up in finite time). This could in principle be balanced by a positive energy density appearing on the right hand side of (2.11).

For the above singular solutions the adiabatic approximation which went into their derivation breaks down, and so we should not trust them near the singularity. Even so, one would expect that there should be a solution for any reasonable initial condition, if we believe in completeness of string theory. This leads us to expect that there should be an exact conformal theory which asymptotically reproduces Mueller's solutions. For the case $N=1$ we will now see directly that this is indeed the case. In fact the corresponding conformal theory turns out to be the $S L(2, R) / U(1)$ coset model which was recently linked to the black hole geometry in [15].

For $N=1$ we have $q_{1}= \pm 1$. Let us take the plus sign for $q$ as the other sign (giving the dual solution [5]) corresponds simply to shifting of time. We thus have

$$
\varphi=\varphi_{0}-\ln \sin 2 b t, \lambda=\lambda_{0}+\ln \tan b t, \quad b^{2}=4 .
$$


This background describes a universe with one spatial dimension with the metric given by (we fix a particular value for $\lambda_{0}$ and assume that $x$ is periodic with period $r$, e.g. $2 \pi$ )

$$
d s^{2}=-d t^{2}+\mathrm{e}^{2 \lambda} d x^{2}=-d t^{2}+b^{-2} \tan ^{2} b t d x^{2}
$$

The universe starts at $t=0$ with zero size and grows to infinite size at $t=\pi / 2 b$. Let us change our coordinates. Let

$$
u=\sin b t \mathrm{e}^{x}, \quad v=\sin b t \mathrm{e}^{-x} .
$$

We find that

$$
d s^{2}=-b^{-2} \frac{d u d v}{1-u v}
$$

and that the unshifted dilaton field $\phi$ is given by

$$
2 \phi=\varphi+\lambda=2 \phi_{0}-2 \ln \cos \mathrm{bt}=2 \phi_{0}-\ln (1-\mathrm{uv})
$$

This is the same metric and dilaton background found in [15] in the region $0 \leq u v \leq 1$ (here $u v=\sin ^{2} b t$ ), i.e. the region between the horizon and singularity. There is one difference though: here we are working on a periodic space, so $x$ is identified with $x+r$. This means that we have to identify

$$
(u, v) \sim\left(\alpha u, \alpha^{-1} v\right)
$$

i.e. we get a wedge in the region between horizon and singularity (see fig. 2). Of course the system of equation we looked at is also valid for infinite radius $r$, which implies that we can in fact reproduce the full region between horizon and singularity with no identification. In this case in order to complete the metric we will have to add regions $I$ and $I I I$ as well, and we end up getting the full black hole solution. It is amusing that the transformation (4.2) is an exact symmetry of the conformal theory (in an appropriate basis it corresponds to conjugating $S L(2, R)$ group element by the diagonal matrix $\left.\left(\alpha^{1 / 2}, \alpha^{-1 / 2}\right)\right)$. So we can consider an infinite orbifold of $S L(2, R) / U(1)$ by the group generated by this symmetry 
and obtain the compact universe solution. One should note that since $u=v=0$ is a fixed point of this transformation we end up getting a new singularity at $u=v=0$, which in our cosmological interpretation corresponds to the initial time $t=0$, where the universe has zero radius. There is another wedge branch of the solution in regions $I$ and $I I I$ which has periodic time which touches the cosmological region at the singular point $u=v=0$.

Note that in this language the duality of conformal theory [17, 21] corresponds (for appropriate choice of radius) to the standard $a \rightarrow 1 / a$ duality of our one dimensional universe, i.e. $b t \rightarrow \frac{\pi}{2}-b t$ changes $u v \rightarrow 1-u v$ and $\lambda \rightarrow-\lambda$.

Another aspect of Mueller's solution is that we can continue time past the singularity at $t=\pi / 2 b$. In particular, if we introduce

$$
u_{1}=-\sin b t \mathrm{e}^{x}, v_{1}=-\sin b t \mathrm{e}^{-x}
$$

we get an identical copy of the interior region of black hole (on the overlap $u_{1}=-u, v_{1}=$ $-v$ ) where the universe now starts at the lower singularity, and evolves till $t=\pi / b$ at which it reaches zero size, it grows as $t$ increases until it becomes infinite in size again at $t=3 \pi / 2 b$. Then again we use the coordinate transformation

$$
u_{2}=\sin b t \mathrm{e}^{x}, v_{2}=\sin b t \mathrm{e}^{-x}
$$

where the radius shrinks to zero size at $t=2 \pi / b$. By now the universe has undergone two oscillations, but we have covered $S L(2, R)$ only once. For example, if we take $x=0$, the corresponding element of $S L(2, R)$, as we evolve in time, is represented by the matrix

$$
\left(\begin{array}{cc}
\cos b t & \sin b t \\
-\sin b t & \cos b t
\end{array}\right)
$$

(it is known that the $S L(2, R) / U(1)$ has two copies of the interior region of black hole-to get only one we could have used $P S L(2, R) / U(1))$. But now we will not identify $\left(u_{2}, v_{2}\right)$ with $(u, v)$, as that would have given us periodic time. Instead we continue forever. This means that in the above matrix realization of $S L(2, R)$ we are not identifying $t \rightarrow t+2 \pi / b$. 
In other words the cosmological interpretation suggests that we go to an infinite fold cover of $S L(2, R)$. In this way we end up with a universe which had no beginning and no end, and it undergoes infinitely many oscillations. Again in this picture we have the option of choosing a finite radius or infinite radius for our space. If we have a finite radius, then we will have to restrict to the wedge region between the horizon and singularity shown in fig.2, otherwise we will have to add the regions $I$ and $I I I$ to complete the metric. In this latter interpretation an observer in region $I$ or $I I I$ of one of these universes, may decide to enter the next universe. The Penrose diagram for this series of universes is shown in fig.3. It is amusing to note that in the Euclidean version of each of these universes we have a periodic coordinate which suggests a thermal bath interpretation. This suggests, even in the cosmological picture, we get radiation from the horizons. It would be interesting to study this further.

Now we ask the question if this one dimensional cosmological solution should be taken seriously. Naively the answer would be no, because we have violated the assumption of adiabaticity of the fields near the singularity, when the size of the universe is infinitely large. However, since we know there is an exact conformal theory with the correct asymptotic behavior, we are led to expect that there exist a corrected metric which gives the exact answer. Based on comparison with the coset model an exact metric for the Euclidean black hole was suggested in [17]. As was checked in [22] the corresponding metric - dilaton background solves the $\sigma$ model conformal invariance conditions in the 3-loop approximation. When one analytically continues the conjectured metric to the region between horizon and singularity it reads

$$
d s^{2}=-d t^{2}+b^{-2} \frac{\tan ^{2} b t}{1+q \tan ^{2} b t} d x^{2} \quad, \quad q=2 / k=8 / 9
$$

If this metric is indeed exact, it suggests that the space-time is actually nonsingular at $t=\pi / 2 b$ so that we see no singularity in the metric! In the finite radius scenario (where we introduce an additional singularity at $u=v=0$ ) we have a universe starting at zero 
radius, reaching a finite maximum radius at finite time and then contracting back to zero radius and continuing this indefinitely. The conjectured form for dilaton $\varphi$ is unchanged from the leading approximation and is thus still singular at $t=\pi / 2 b$. The cosmological interpretation of the black hole gets rid of a puzzling consequence of this conjectured exact metric: if analytically continued to the region behind black hole singularity the metric becomes Euclidean between $1 \leq u v \leq 9$ with a singularity at $u v=9$. This is avoided in our interpretation because the region beyond the singularities are never reached (they are connected to universes labeled by $\pm \infty)$.

It would be interesting to find exact conformal field theory generalisations of higher dimensional Mueller-type cosmological solutions 8 . The coset theories like $S O(d, 1) / S O(d-$ 1,1) which should be analogs of the (anti) DeSitter backgrounds (see Bars [21] and refs. there) does not, however, correspond to a flat $N$-space. The generalization of the leading order conformal invariance equations to the case of isotropic homogeneous metric with a curved space (with positive, zero or negative curvature $k$ ) is given by (cf. (3.1)-(3.3))

$$
\begin{gathered}
c-N \dot{\lambda}^{2}+\dot{\varphi}^{2}=-k N(N-1) \mathrm{e}^{-2 \lambda}, \\
\ddot{\lambda}-\dot{\varphi} \dot{\lambda}=-k(N-1) \mathrm{e}^{-2 \lambda}, \\
\ddot{\varphi}-N \dot{\lambda}^{2}=0
\end{gathered}
$$

(the absence of the correction in the third equation is due to the fact that the "potential" in the present case is "classical", i.e. does not depend on the dilaton).Let us assume that $c<0, k>0$. Then at large negative $\lambda$ (small times) the solution is approximately given by the DeSitter metric and a constant dilaton $\phi=\frac{1}{2}(\varphi+N \lambda)$. In this limit, however, the

\footnotetext{
8 In such a case it would be natural to allow the full moduli of toroidal compactifications to become involved. The picture is a more or less straight-forward generalization of the equations we have considered, and we would simply have to study geodesic equations on the corresponding moduli spaces of toroidal compactifications.
} 
adiabatic approximation is not fully reliable. At large positive $\lambda$ the effect of the space curvature becomes irrelevant and the solution approaches the Mueller's isotropic solution

$$
\lambda=\lambda_{0}+N^{-\frac{1}{2}} \ln \tanh b t \rightarrow \text { const }, \varphi \rightarrow-\text { bt } .
$$

So the universe is born at $t=0$ as a $d=N+1$ DeSitter space and evolves into the product of a time line and an $N$-sphere at large $t$. This appears to be a direct higher dimensional (Minkowski signature) analog of the $d=2$ (euclidean) "cigar" metric.

So far we have discussed non-critical string cosmology in absence of matter. It is natural to ask how can one introduce 'thermal' non-critical matter, in order to have a richer non-critical string cosmology. It turns out that this can be done at least formally. The usual way we consider thermal ensemble in field theory is by making the time Euclidean with period $\beta$. For non-critical strings the Liouville field plays the role of time and this suggests making it Euclidean and periodic with period $\beta$. The computation of thermal free energy, which is a one loop computation generalizing that of [23] can be carried out explicitly for the non-critical strings $c_{m} \leq 1$. For $c_{m}=1$ noncritical strings at radius $a$, for example, one finds that the necessary modular integral has been evaluated in [24] in a completely different context. This is related to the $S^{1} \times S^{1}$ partition function integrated over the fundamental domain and turns out to be the same as that for the critical $N=2$ strings partition function [25] ( or, equivalently, for $c_{m}=1$ non-critical $N=2$ string partition function). One finds for the free energy

$$
\beta F=\ln \left[\tau_{2} \eta^{2}(\tau) \bar{\eta}^{2}(\bar{\tau})\right]+(\tau \rightarrow \rho)
$$

where $\tau=i \tau_{2}=i \beta a / 8 \pi, \rho=i \beta / 8 \pi a$ and $\eta$ is the Dedekind eta function. Solving the adiabaticity condition one can obtain the energy $E(a)$ from the above expression for $F$. For large entropy and small $a$ we have

$$
E(a)=\frac{A}{a+a^{-1}}
$$


where $A$ depends on the entropy. The profile for $E(a)$ is very similar to that of critical strings (fig.1) except that there is no flat Hagedorn region and we only get a maximum at $\lambda=0$. This is to be expected since at $c_{m}=1$ there is no exponential degeneracy of states, as there is only one massless scalar (and some additional discrete states [26]) and so there is no limiting temperature. In fact it has been suggested [27] that introduction of temperature for non-critical strings might lead to a more clear phase diagram picture for non-critical strings. Note that for large $a, E(a)$ behaves like the energy for that of a massless particle $(E \propto 1 / a)$. For yet larger radii, the temperature drops to zero, but $E$ does not go to zero. It becomes negative and behaves as

$$
E=-\frac{1}{24}\left(a+\frac{1}{a}\right)
$$

This is a Casimir - type contribution which is independent of the temperature. It is in accord with the result of [23] which corresponds to computing the free energy at zero temperature. Note that this Casimir effect is absent in critical (super)-strings, as the cosmological constant vanishes at one loop. Putting the potential $E(a)$ on the right hand side of our equations will modify Muellers solution. The intuition based on the $\lambda$ rolling on the potential hill $E(a)$ implies that the solutions are still singular (and blow up in finite time).

These ideas can be applied to $c_{m}<1$ as well. In this case we have a one dimensional field theory (corresponding to the Liouville field), which we can identify with a space coordinate $x$. The relevant equation to solve for the dilaton at the tree level is $c-\varphi^{\prime 2}=0$ which gives rise to the familiar linear dependence of the dilaton: $\varphi=-\sqrt{c} x$. However, the one loop partition function [23] modifies the above equation to

$$
c-\varphi^{\prime 2}=-h \mathrm{e}^{\varphi}
$$

where $h$ is a positive constant depending on which minimal model we are dealing with. Solving the above equation we find

$$
\varphi=\varphi_{0}-\ln \sinh ^{2} \frac{\sqrt{c} x}{2} .
$$

This suggests a truncation of the Liouville space to a half-line. Again we are going beyond the validity of adiabatic approximations but we are getting the hint that the linear dependence of dilaton is modified at one loop. 


\section{Conclusions}

We have discussed some aspects of string cosmology taking into account the dynamics of the dilaton field which is needed to restore the duality symmetry in string theory. We have considered both critical and non-critical strings, the non-critical strings being the analog of having a non-vanishing cosmological constant in the Einstein theory.

For critical strings we find a major modification of the description of the early universe. In particular, winding string states, if not annihilated, can halt the expansion of the universe. This is against the intuition based on standard gravitational theory where a constant energy density leads to inflation. We have seen that string dynamics is consistent with the following picture of cosmology which needs to be verified. A nine dimensional spatial universe of Planck size which we take to be a periodic "box" , at the Hagedorn temperature, expands in all directions. Not being able to get rid of winding modes the expansion stops and the universe contracts to even smaller than the Planck scale. Now the momentum modes cannot annihilate one another and the contraction stops. The universe starts to grow once again. The universe oscillates for a while in this fashion. In this way one can in principle generate a lot of entropy. Fluctuations cause different directions to expand at different times. One of these fluctuations leads to three dimensions expanding, and now the winding modes can get annihilated and the universe expands, getting rid of the winding modes. As the temperature drops, the Planckian string states get suppressed by the Boltzman factor and we are left with the massless modes in a three dimensional expanding universe, which looks very much like the radiation dominated era of the standard cosmology. It is remarkable that the radiation dominated era of the standard cosmology emerges with no fine tuning.

How can one test this picture? One idea is to treat strings classically as one does for cosmic strings. So we can study the evolution of a universe filled with these strings which interact with each other by cutting and rejoining. The main difference with the cosmic strings is that we have a different evolution equation for the universe, and the coupling 
constant for the cutting and rejoining which is related to dilaton field is also evolving. This approach will presumably answer questions of entropy production and homogeneity of the early universe in the cosmological scenario described above.

We have also studied non-critical string cosmologies, which seem qualitatively rather different from the critical string case. In particular we have related the empty universe cosmology in $d=2$ to the two dimensional 'black hole' solution (more precisely to its universal cover). Could it be that stringy black-holes in $4 \mathrm{~d}$ behave in a similar fashion? In particular, as we cross the horizon we enter another universe instead of encountering the singularity? It would be remarkable if the "cosmology-black hole" connection discovered in two dimensions has higher dimensional analogs. Indeed, the fact that the singularity of standard $4 \mathrm{~d}$ black hole solution is also spacelike, i.e. appears at a given time rather than space, suggests a cosmological interpretation.

To discuss non-critical string cosmologies with matter we have to introduce the notion of temperature for non-critical strings. We have made a suggestion of how this may be done (by a formal wick rotation of Liouville field and making it periodic with period $\beta$ as one does for $c_{m}=25$ in order to define critical string thermodynamics $)$. 6 . Using this definition we have computed the thermal properties of $d=1$ matter (which can be easily generalized to $d<1$ as in [23]). It is an interesting question to see if there exists a matrix model analog of this finite temperature interpretation (for example are unitary matrix models related to thermalizations of standard matrix theories?).

We would like to thank M. Bershadsky, A. Guth, S. Jain, J. Polchinski, M. Tsypin, G. Veneziano and E. Verlinde for interesting discussions. A.T. would like to acknowledge J.Bagger for hospitality at Johns Hopkins University and various kinds of help. The research of A.T. was supported by NSF grant PHY-90-96198 and that of C.V. was supported in part by Packard Foundation and NSF grants PHY-89-57162 and PHY-87-14654.

9 At higher genus due to the coupling of Liouville field to the curvature, this may be consistent only if $\beta$ is quantized. 


\section{Appendix A.}

In this appendix we discuss the solution to the equations $(3.1),(3.2),(3.3)$ with $c=0$ and

$$
E \propto \exp (\alpha \lambda)
$$

starting with initial conditions $\dot{\varphi}<0, \dot{\lambda}>0$. In particular we show that if the total energy grows with volume, i.e., if $\alpha>0$ the universe stops expanding in finite time and starts contracting, i.e., we reach $\dot{\lambda}=0$ in finite time as $\lambda$ is decelerating. If $\alpha<0$ the universe expands forever and if $\alpha=0$ the universe comes to a halt, without turning back. We will show in addition that if $\alpha=-1$, as would be the case for radiation dominated era, the radius eventually grows as in standard Einstein's gravity in the radiation dominated era.

One can reach the above conclusions more or less immediately by using the ideas developed in section 3. As discussed there starting with $\dot{\varphi}<0$, we conclude that $\dot{\varphi}$ cannot become zero, and thus it will always remain negative. Since $\ddot{\varphi}>0$ from (3.3) we conclude that $\dot{\varphi}$ approaches zero and gets there at $t=\infty$. Equation (3.2) implies that we can treat $\lambda$ as the position of a particle rolling on a potential described by $\mathrm{e}^{\varphi} E(\lambda) / 2 N$ with a damping term (because of $\dot{\varphi}$ terms in (3.2)). The potential is getting weaker as its overall size is modulated by $\mathrm{e}^{\varphi}$ which is decreasing because $\dot{\varphi}<0$ and the damping term gets weaker as $\dot{\varphi}$ is approaching zero. For $\alpha<0$ it is clear that since $E$ is decreasing with $\lambda, \lambda$ will continue to increase indefinitely. For $\alpha>0$, since the energy increases with $\lambda$, the universe cannot grow indefinitely, and will reach a maximum and start contracting. This is almost obvious, were it not for the fact that the energy is modulated with $\mathrm{e}^{\varphi}$ which is getting smaller. So the universe could conceivably continue expanding at ever slower rates. This we will show is not the case. We shall find by a careful analysis of these equations that the universe reaches a maximum size and starts contracting after that. The fact that for $\alpha=0$ (i.e., constant energy), the universe reaches a maximum size and stops is also clear from (3.2) as the potential is flat in this case, and the damping term will eventually stop $\lambda$. 
First we note that $P=-N^{-1} \partial E / \partial \lambda=-\alpha E / N$. Then we can eliminate $\mathrm{e}^{\varphi} E$ from equations (3.1), 3.2), (3.3) and obtain

$$
\begin{aligned}
& \ddot{\lambda}-\dot{\varphi} \dot{\lambda}=\frac{-\alpha}{2 N}\left(-N \dot{\lambda}^{2}+\dot{\varphi}^{2}\right), \\
& \ddot{\varphi}-N \dot{\lambda}^{2}=\frac{1}{2}\left(-N \dot{\lambda}^{2}+\dot{\varphi}^{2}\right) .
\end{aligned}
$$

Now we can represent these equations in the first order form by defining

$$
l=\dot{\lambda}, \quad f=\dot{\varphi}
$$

to obtain

$$
\begin{gathered}
i=\frac{\alpha l^{2}}{2}+l f-\frac{\alpha f^{2}}{2 N}, \\
\dot{f}=\frac{N l^{2}}{2}+\frac{f^{2}}{2} .
\end{gathered}
$$

The initial conditions are $f<0$ and $l>0$. Actually we have another restriction, which comes from positivity of $E$ in (3.1)

$$
|f| \geq|\sqrt{N} l|
$$

The important point to note in solving (A.2) is that these equations are homogeneous. In other words, if we rescale $(l, f) \rightarrow r(l, f)$ and rescale $t \rightarrow r^{-1} t$ we get a new solution. This means, in particular, that if we look for solutions with

$$
\frac{d f}{d l}=\frac{f}{l}
$$

we get straight lines in the $(f, l)$ plane which pass through $(0,0)$. Studying these particular solutions will give us a handle to study a qualitative behavior of all solutions. These straight line solutions can be easily found by noting that $d f / d l=\dot{f} / i$. Solving (A.3) we find three solutions

$$
\frac{f}{l}= \pm \sqrt{N}, \frac{N}{\alpha}
$$


By the rescaling argument, $f$ and $l$ can approach zero only along the above lines. So in the region $\alpha>0$, if we start with the initial conditions $l>0, f<0,|f|>\sqrt{N} l$, the only possibility for not crossing the $l=0$ line is for the flow to approach zero through the line $f / l=-\sqrt{N}$. But a simple check of the equation shows that this line is repulsive, i.e., the flows are driven away from it. They will be attracted to the next fixed line which is in the $l<0$ region. They will cross the $l=0$ line which means that at finite time $\dot{\lambda}=0$. According to (3.2) $\ddot{\lambda}$ is not zero so the universe bounces back and $\lambda$ starts to decrease.

For $\alpha=0$, the solution with $l=0$ is an attractive solution and all solutions will approach it. This simply means that if we start with $\dot{\lambda}>0$ the expansion will eventually stop because of the damping term. In fact, it is easy to write the general solution in this case: using (2.11) and (3.2) ( with $P=0$ ) we get

$$
\mathrm{e}^{-\varphi}=\frac{E_{0} t^{2}}{4}-\frac{N A^{2}}{E_{0}}, \lambda=\lambda_{0}+\frac{1}{\sqrt{N}} \ln \frac{t-2 \sqrt{N} A / E_{0}}{t+2 \sqrt{N} A / E_{0}} .
$$

This solution approximately describes the evolution in the Hagedorn region (in fact, $E$ is not quite constant and decreases with increasing $\lambda$ so the expansion never quite stops).

$$
\text { If }-\sqrt{N}<\alpha<0 \text {, the line } f / l=N / \alpha \text { is an attractive solution and all solutions will }
$$
approach it. Note that for $\alpha=-1$ which corresponds to the case of the radiation dominated era, the fixed line is $f / l=-N$. For the fixed line solution $2 \dot{\phi}=\dot{\varphi}+N \dot{\lambda}=f+N l=0$, i.e. the original dilaton $\phi$ is constant and we get the standard (flat) FRW - type cosmology in the radiation dominated era, $a \sim t^{2 /(N+1)}$. An important point is that this is an attractive solution, i.e. all solutions (with initial $\dot{\varphi}<0$ ) will eventually approach it ! So at late times we get the standard radiation dominated era of cosmology without any fine tuning. If $\alpha<-\sqrt{N}$ the attractive solution will be $f / l=-\sqrt{N}$. 


\section{References}

1. K. Kikkawa and M. Yamasaki, Phys. Lett. B149 (1984) 357;

N. Sakai and I. Senda, Progr. Theor. Phys. 75(1986)692;

V. Nair, A. Shapere, A. Strominger and F. Wilczek, Nucl. Phys. B287(1987)402;

B. Sathiapalan, Phys. Rev. Lett. 58 (1987) 1597.

2. R. Brandenberger and C. Vafa, Nucl. Phys. B316(1988) 391.

3. A.R. Bogojevic, Brown Univ. preprint 1988, BROWN-HET-691;

E. Witten, Phys. Rev. Lett. 61(1988)670 ;

A.A. Tseytlin, Nucl. Phys. B350(1991)395.

4. P. Ginsparg and C. Vafa, Nucl. Phys. B289(1987)414;

T.H. Buscher, Phys. Lett. B194(1987)59 ; Phys. Lett. B201(1988)466;

T. Banks, M. Dine, H. Dijkstra and W. Fischler, Phys. Lett. B212(1988) 45;

G. Horowitz and A.A. Steif, Phys. Lett. B250(1990)49;

E. Smith and J. Polchinski, Phys. Lett. B263(1991)59.

5. A.A. Tseytlin, Mod. Phys. Lett. A6(1991)1721.

6. G. Veneziano, preprint CERN-TH-6077/91.

7. A.A. Tseytlin, "Space-time duality, dilaton and string cosmology", Proc. of the First International A.D. Sakharov Conference on Physics, Moscow 27- 30 May 1991, ed. L.V. Keldysh et al., Nova Science Publ., Commack, N.Y., 1991.

8. H. Nishimura and M. Tabuse, Mod. Phys. Lett. A2(1987)299;

J. Kripfganz and H. Perlt, Class. Quant. Grav. 5(1988)453;

N. Matsuo, Z. Phys. C36(1987)289;

Y. Leblanc, Phys. Rev. D10(1988)3087;

9. M. Hellmund and J. Kripfganz, Phys. Lett. B241(1990)211.

10. N. Sanchez and G. Veneziano, Nucl. Phys. B333(1990)253;

B.A. Campbell, A. Linde and K.A. Olive, Nucl. Phys. B355(1991)146. 
11. M.C. Bento, O. Bertolami and P.M. Sa, Phys. Lett. B262(1991)11 .

12. R. Myers, Phys. Lett. B199(1987)371 .

13. I. Antoniadis, C. Bachas, J. Ellis, D. Nanopoulos, Phys. Lett. B211(1988)393; Nucl. Phys. B328(1989)115.

14. M. Mueller, Nucl. Phys. B337(1990)37.

15. E. Witten, Phys. Rev. D44(1991)314.

16. S. Elitzur, A. Forge and E. Rabinovici, Nucl. Phys. B359(1991)581;

G.Mandal, A.M. Sengupta and S.R. Wadia, Mod. Phys. Lett. A6(1991)1685.

17. R. Dijgraaf, H. Verlinde and E. Verlinde, Princeton preprint PUPT-1252/91.

18. J. Scherk and J.H. Schwarz, Nucl. Phys. B81(1974)118;

E.S. Fradkin and A.A. Tseytlin, Nucl. Phys. B261(1985)1;

C.G. Callan, D. Friedan, E. Martinec, M.J. Perry, Nucl. Phys. B262(1985)593.

19. N. Deo, S. Jain and C.-I. Tan, Phys. Rev. D40(1989)2626 ;

Phys. Lett. B220(1989)125; Harvard preprint HUTP-91/A025.

20. M. Bowick and S. Giddings, Nucl. Phys. B325(1989)631 .

21. A. Giveon, LBL preprint LBL-30671/1991;

E. Kiritsis, LBL preprint LBL-30747/1991;

I. Bars, U. of Southern California preprint USC-91/HEP-B3 (1991).

22. A.A. Tseytlin, Phys. Lett. B268(1991)175.

23. D.J. Gross and I.R. Klebanov, Nucl. Phys. B344(1990)475;

M. Bershadsky and I.R. Klebanov, Phys. Rev. Lett. 65(1990)3088;

N. Sakai and Y. Tanii, Int. J. Mod. Phys. A6(1991)2743.

24. L. Dixon, V. Kaplunovsky and J. Louis, Nucl. Phys. B355(1991)649.

25. H. Ooguri and C. Vafa, Nucl. Phys. B361(1991)469.

26. D.J. Gross, I.R. Klebanov and M.J. Newman, Nucl. Phys. B350(1991)621;

A.M. Polyakov, Mod. Phys. Lett. A6(1991)635.

27. C. Vafa, Int. Jour. of Mod. Phys. A6(1991)2829. 


\section{Figure Captions}

Fig. 1. The solid curve represents the adiabatic variation of energy $E$ as a function of scale $\lambda$. Note the duality symmetry $\lambda \rightarrow-\lambda$. Near the Planck scale $(\lambda \sim 0)$ the energy is more or less independent of scale, as we are in the Hagedorn regime of string thermodynamics. For large radii, we enter the radiation dominated era and $E \propto \exp -\lambda$. The dashed curve represents the effective energy if the winding modes (and momentum modes for $\lambda<<0$ ) fail to annihilate.

Fig. 2. The 2d black hole geometry is represented here. The cosmological solution correspond to regions $I I$ and $I V$. If we wish to deal with a compact universe, we are limited to the wedge drawn here. In this case the curve drawn in region $I I$ inside the wedge represents the space, with its endpoints identified.

Fig. 3. The 'accordion-like' Penrose diagram of $2 \mathrm{~d}$ cosmology. An observer in universe $n$ can end up in any universe $m \geq n$. The regions inside the curves represent the universe if we wish to consider compact space. 